\title{
Evaluation of a new rapid test for the detection of influenza $A$ and $B$ viruses and pandemic (H1N1) 2009 virus subtyping in respiratory samples
}

The emergence in 2009 of a novel influenza A (H1N1) virus was responsible for a pandemic outbreak of febrile respiratory infections worldwide (NSOIAVIT, 2009). During the first pandemic wave, specific real-time RT-PCR (rRT-PCR) assays were the reference methods for the detection of the pandemic (H1N1) 2009 influenza virus, leading to the overload of laboratory facilities due to the large number of clinical samples analysed (Duchamp et al., 2010). The re-emergence during winter 2011 of the pandemic (H1N1) 2009 virus highlighted the need for commercially available and readily performed influenza diagnostic tests capable of detecting and subtyping influenza A viral strains in respiratory samples. The previously reported sensitivity of commercially available rapid influenza antigen tests (RIATs) for pandemic (H1N1) 2009 virus was 11 to $80 \%$, and generally evaluated at around $60 \%$ for clinical specimens (Drexler et al., 2009; Lee et al., 2010; Lévêque et al., 2010; Louie et al., 2010). Moreover, currently available RIATs are only able to type influenza A and B viruses, and do not specifically subtype the pandemic (H1N1) 2009 virus. The aim of this study was thus to assess the diagnostic performances, comparatively to the rRTPCR assay, of a new RIAT, the ONE STEP influenza virus $\mathrm{A} / \mathrm{B} / \mathrm{A}(\mathrm{H} 1 \mathrm{~N} 1)$ pandemic rapid test (Standard Diagnostics; Bioline), which can detect the influenza viruses $A$ and B, and subtype the pandemic (H1N1) 2009 in respiratory samples.

One hundred and six nasal swab specimens collected in SeptemberDecember 2009 from patients of all age groups with influenza-like illness were retrospectively tested by RIAT. The Virocult system (Lakewood Biochemical), consisting of a rayon-budded swab and a transport tube of liquid virus transport medium, was used. Among the 106 clinical samples investigated, 56 had previously tested positive for pandemic (H1N1) 2009 influenza virus by $\mathrm{H} 1$ specific $\mathrm{rRT}-\mathrm{PCR}$ assay (Duchamp et al., 2010). A total of 40 samples had previously tested positive for seasonal influenza A virus [10 (H1N1) and 20 (H3N2) influenza A virus specimens] or influenza $B$ virus (10 specimens) by influenza A and B specific rRT-PCR assays developed by the French National Reference Centres for influenza viruses. Ten samples negative by rRT-PCR assays for influenza A and B viruses were also randomly selected. Nasal swab samples were rapidly transported to the virology laboratory (Reims University Medical Centre, Reims, France) where $500 \mu$ l virus transport medium was added to the Virocult system, then each sample was divided between two sterile tubes. The first aliquot was prospectively tested for pandemic and seasonal influenza A and B viruses, while the second aliquot was used for the retrospective RIAT analysis. The ONE STEP influenza virus $\mathrm{A} / \mathrm{B} / \mathrm{A}(\mathrm{H} 1 \mathrm{~N} 1)$ pandemic rapid test is a chromatographic immunoassay with strips with immobilized mouse monoclonal anti-A(H1N1) pandemic haemagglutinin, anti-influenza A and anti-influenza B nucleoprotein antibodies. The RIAT was carried out according to the manufacturer's instructions using $100 \mu \mathrm{l}$ of the second aliquot stored at $-80{ }^{\circ} \mathrm{C}$.

Overall, from the 56 specimens positive for the pandemic (H1N1) 2009 influenza virus, the new rapid antigen test detected 22 positive samples, demonstrating a sensitivity of $39.3 \%$ (range $26.5-52.1 \%$ ).

This appeared to be lower than the sensitivity range $68.5-77 \%$ recently reported in similar studies testing the same RIAT with the same sampling technique in comparison with a rRT-PCR assay (Choi et al., 2010; Kwon et al., 2011). A potential effect of the storage at $-80{ }^{\circ} \mathrm{C}$ on the target antigens detected by the RIAT could not be ruled out, in particular in cases of low viral loads, as an explanation for the moderate sensitivity observed. No false-positive result for pandemic (H1N1) 2009 influenza virus detection was observed when seasonal influenza A and B virus-positive and negative samples were tested with the new RIAT, indicating a specificity and a positive predictive value of $100 \%$. Thirty-four samples positive for the pandemic (H1N1) 2009 influenza virus by rRT-PCR assay were not detected by the RIAT, demonstrating a negative predictive value of $59.5 \%$ (range 46.5-72.6\%). However, among these 34 samples, 4 were positive with the rapid antigen test for influenza $A$ virus but were not identified as a pandemic variant, indicating that results positive for seasonal influenza A virus should be necessarily checked by a reference rRT-PCR assay for influenza A virus subtyping.

The viral load assessed in each influenza A-positive specimen using a rRT-PCR assay targeting the influenza A matrix (M) gene demonstrated that the positivity of the RIAT appeared to be significantly associated (Wilcoxon test) with the pandemic (H1N1) 2009 viral load levels in the clinical samples, since the median values were $5.5 \times 10^{6} \pm 3.9 \times 10^{7}$ and $5.7 \times 10^{4} \pm 6.9 \times 10^{6} \mathrm{M}$ gene copies $\mathrm{ml}^{-1}$ in RIAT positive and negative samples, respectively $\left(P<10^{-3}\right)$ (Fig. 1). A receiver operating characteristics (ROC) analysis was also performed, showing an area under the curve of 0.804 (ROC value range 0.689-0.918) and confirming that the RIAT results were well correlated with the viral load quantified by the M rRT-PCR specific assay in the clinical samples. In this study, the smallest viral load detected in a clinical sample by the RIAT was $3.5 \times 10^{3}$ $\mathrm{M}$ gene copies $\mathrm{ml}^{-1}$. Interestingly, RIAT performances appeared to be better for seasonal influenza A viruses than for pandemic (H1N1) 2009 virus diagnosis, since the median value of seasonal influenza A viral loads in clinical samples positive by 


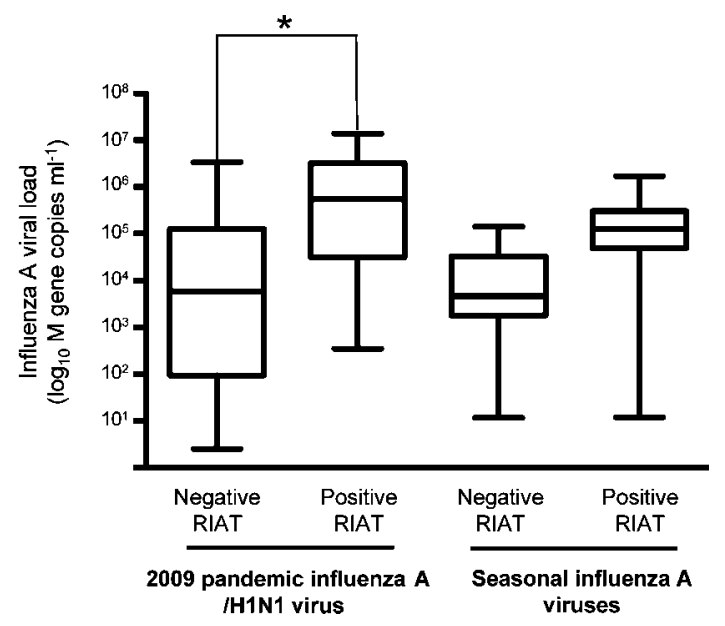

Fig. 1. Box plots showing pandemic and seasonal influenza A viral load levels ( $y$-axis) in the case of positive and negative RIAT results ( $x$-axis). The ends of the boxes are the 25th and 75th quartiles, respectively. The lines across the middle of the boxes identify the median values of the influenza $A$ viral loads in the respiratory specimens tested. The asterisk indicates the statistically significant result.

the RIAT was lower $\left(8.9 \times 10^{5} \pm 2.4 \times 10^{6} \mathrm{M}\right.$ gene copies $\mathrm{ml}^{-1}$ ) than for pandemic (H1N1) 2009 virus (Fig. 1). This could potentially explain the misidentification as seasonal influenza A virus of four samples positive for the pandemic variant. By contrast, Kwon et al. (2011), who developed this new RIAT in collaboration with the Standard Diagnostics Company, did not observe any difference in the detection limit between the haemagglutinin protein of $\mathrm{A} /$ Korea/01/ 2009 and the nucleoprotein of seasonal influenza A viruses when the kit was applied to clinical specimens.

In conclusion, our data showed a limited clinical value of this new rapid antigen test as a first-line screening strategy for nasopharyngeal samples obtained from (H1N1) 2009 virus suspect cases in daily virological diagnosis, since negative or positive results for seasonal influenza $\mathrm{A}$ virus should be necessarily checked by rRT-PCR assay for influenza A virus detection and subtyping.

\section{Acknowledgements}

We are indebted to the technicians who have prospectively and retrospectively tested the nasopharyngeal samples of the present study. This study was supported by a grant for clinical and virological research from the Medical University and School of Medicine of Reims (EA-4303/IFR53) and by the PHRC Gripmask (PHRC 2008, P070160). Fanny Renois is supported by an official grant from the French Army (Bourse DGA, Délégation Générale de l'Armement, Ministère de la Défense; topic microbiology, infectious diseases; 2009-2012).

\section{Nicolas Lévêque, ${ }^{1,2}$ \\ Déborah Talmud, ${ }^{1,2}$ Fanny Renois, ${ }^{1,2}$ Coralie Barbe ${ }^{3}$ and Laurent Andréoletti ${ }^{1,2}$ \\ ${ }^{1}$ Unité de Virologie Médicale et Moléculaire, Centre Hospitalier Universitaire, Reims, France \\ ${ }^{2}$ IFR 53/EA-4303, Faculté de Médecine, Reims, France \\ ${ }^{3}$ Coordination de la Recherche Clinique, Centre Hospitalier Universitaire, Reims, France}

Correspondence: Nicolas Lévêque (nleveque@chu-reims.fr)

Choi, Y. J., Nam, H. S., Park, J. S., Kim, H. J., Park, K. B., Jeon, M. H., Kim, C. J., Hwangbo, Y., Park, K. S. \& Baek, K. A. (2010). Comparative analysis of the multiple test methods for the detection of pandemic influenza A/H1N1 2009 virus. J Microbiol Biotechnol 20, 1450-1456.

Drexler, J. F., Helmer, A., Kirberg, H., Reber, U., Panning, M., Müller, M., Höfling, K., Matz, B., Drosten, C. \& Eis-Hübinger, A. M. (2009). Poor clinical sensitivity of rapid antigen test for influenza A pandemic (H1N1) 2009 virus. Emerg Infect Dis 15, 1662-1664.

Duchamp, M. B., Casalegno, J. S., Gillet, Y., Frobert, E., Bernard, E., Escuret, V., Billaud, G., Valette, M., Javouhey, E. \& other authors (2010). Pandemic A(H1N1)2009 influenza virus detection by real time RT-PCR: is viral quantification useful? Clin Microbiol Infect 16, 317-321.

Kwon, D., Shin, K., Kwon, M., Oh, H. B., Kang, C. \& Lee, J. Y. (2011). Development and evaluation of a rapid influenza diagnostic test for the pandemic (H1N1) 2009 influenza virus. J Clin Microbiol 49, 437-438.

Lee, G. C., Jeon, E. S., Kim, W. S., Le, D. T., Yoo, J. H. \& Chong, C. K. (2010). Evaluation of a rapid diagnostic test, NanoSign $\mathbb{B}$ influenza $\mathrm{A} / \mathrm{B}$ antigen, for detection of the 2009 pandemic influenza $\mathrm{A} / \mathrm{H} 1 \mathrm{~N} 1$ viruses. Virol J 7, 244.

Lévêque, N., Coste, J. F., Renois, F., Brodard, V., De Champs, C. \& Andréoletti, L. (2010). Preliminary evaluation of two rapid antigen assays for novel influenza A (H1N1) virus detection in clinical specimens. $J$ Med Microbiol 59, 508-509.

Louie, J. K., Guevara, H., Boston, E., Dahlke, M., Nevarez, M., Kong, T., Schechter, R., Glaser, C. A. \& Schnurr, D. P. (2010). Rapid influenza antigen test for diagnosis of pandemic (H1N1) 2009. Emerg Infect Dis 16, 824-826.

NSOIAVIT (2009). Emergence of a novel swine-origin influenza A (H1N1) virus in humans. N Engl J Med 360, 2605-2615. Novel Swine-Origin Influenza A (H1N1) Virus Investigation Team. 